\title{
Archives
}

\section{Archives et réemploi dans les films expérimentaux}

\section{Annaëlle Winand}

Volume 46, numéro 1, 2016

URI : https://id.erudit.org/iderudit/1035721ar

DOI : https://doi.org/10.7202/1035721ar

Aller au sommaire du numéro

Éditeur(s)

Association des archivistes du Québec (AAQ)

ISSN

0044-9423 (imprimé)

2369-9256 (numérique)

Découvrir la revue

Citer cet article

Winand, A. (2016). Archives et réemploi dans les films expérimentaux. Archives, 46(1), 35-45. https://doi.org/10.7202/1035721ar

\section{Résumé de l'article}

Dans son article, Annaëlle Winand nous expose à l'utilisation des archives par les artistes de réemploi expérimentaux dans les films et les vidéos. En faisant un survol de la littérature contemporaine sur le sujet, l'auteure cherche à identifier les préoccupations des chercheurs. Quel intérêt les artistes portent-ils aux documents d'archives ? Quels enjeux sont liés à la mixité du cinéma et des archives ? L'auteure évoque d'abord l'atteinte d'un consensus sur la définition de que l'on entend par le concept d'" archive ». Elle fait aussi état d'une structure d'analyse commune à la littérature sur le sujet : la temporalité, l'historicité et la mémoire, toutes trois communes aux archives et au cinéma. Enfin, elle insiste sur la nécessité de la collaboration entre artistes, archivistes et chercheurs, afin de comprendre l'archive en elle-même, mais aussi les problématiques, typiquement pratiques, et reliées à son utilisation, telle que la préservation, la conservation ou l'accès et ce, particulièrement à l'ère du numérique.

L'utilisation des archives dans les films par les artistes de réemploi expérimentaux est particulièrement intéressante parce que ces derniers voient les documents d'archives en dehors de leur contexte et savent les interpréter sous un nouveau jour.
Ce document est protégé par la loi sur le droit d'auteur. L'utilisation des services d’Érudit (y compris la reproduction) est assujettie à sa politique d'utilisation que vous pouvez consulter en ligne.

https://apropos.erudit.org/fr/usagers/politique-dutilisation/ 


\section{| ÉTUDE \\ Archives et réemploi dans les films expérimentaux}

\section{Annaëlle Winand}

\section{INTRODUCTION}

Dans le cadre de notre recherche de doctorat, nous nous intéressons à l'utilisation d'archives et de documents audiovisuels (considérés comme archives) par les artistes de réemploi expérimentaux dans les films et vidéos. Bien que peu exploré dans les travaux archivistiques, il s'agit pourtant d'un phénomène bien connu des études cinématographiques. L'historien et cinéaste Jay Leyda (1964) est le premier théoricien à attribuer le nom de compilation film à des films créés à partir de morceaux de films préexistants. Il en repère les premiers usages dès les débuts du cinéma et principalement dans le cadre de la production de documentaires. Cette pratique va ensuite se développer et atteindre les artistes issus de l'avant-garde avec les procédés de collage dans les années 1920 (Wees 2014). Mais, c'est dans les années 1950-1960 que les pratiques de réemploi vont vraiment émerger dans le cinéma expérimental (Habib 2008).

La thématique du cinéma de réemploi est particulièrement étudiée depuis le milieu des années 1990. Il est d'ailleurs possible de faire remonter cet engouement particulier pour les films faits d'archives à la publication de l'ouvrage Recycled Images: The Art and Politics of Found Footage (Wees 1993). Pour plusieurs chercheurs, dont André Habib (2014), cet enthousiasme critique et théorique s'inscrit dans le centenaire du cinéma, coïncidant avec la révolution du numérique, tous deux porteurs de questionnement sur la nature du film, des archives filmiques et du réemploi (Cammaer 2014).

Plus récemment, le cinéma de réemploi a connu un important regain d'intérêt dans les études cinématographiques, comme l'a souligné Christa Blümlinger (2014a). Une littérature riche sur la question du réemploi, des archives et du cinéma a en effet fait son apparition depuis 2010 en Amérique du Nord principalement, mais aussi en Europe.

Dans ce présent travail, nous tenterons donc de relever les préoccupations des chercheurs, à partir de ces récents textes. Il s'agira d'une première étape pour comprendre, du point de vue des études cinématographiques, les préoccupations contemporaines et l'état de la recherche en matière de cinéma et d'archives, dans le cadre précis du réemploi expérimental. 


\section{Archive, archives, contre-archive, beretical archive et anarchive}

En parcourant les ouvrages sur le thème du réemploi, nous constatons une grande diversité des termes utilisés pour désigner l'archive. Comme le souligne Anne Klein dans sa thèse de doctorat (2015), le concept d'archive est en effet employé dans les champs artistique et philosophique sous différentes formes : il est tour à tour question des documents eux-mêmes, de leur structure, de leur rapport au passé et à la mémoire ou encore des méthodes d'organisation qui les constituent. Les auteurs font dès lors souvent référence à l'archive, au singulier, potentiellement dotée de ces multiples significations, recouvrant ou non les archives, au pluriel, se rapportant généralement aux documents d'archives.

La notion d'archive employée par les études cinématographiques est également chargée de cette signification. Dans la littérature, les principales références en matière d'archive proviennent des textes Archéologie du savoir de Michel Foucault (1969) et Mal d'archive: une impression freudienne de Jacques Derrida (1995). À partir des définitions proposées par ces philosophes, invitant à une certaine interdisciplinarité, plusieurs notions sont développées autour de l'archive. Il est question dans la littérature de contre-archive (Tebo 2010), d'anarchive (Derrida 1995), d'archive vivante (Blümlinger 2014a) ou encore d'beretical archive (Torlasco 2013). L'archive n'est dès lors pas précisément définie par les auteurs, mais semble plutôt s'adapter aux contextes d'analyse.

Ainsi, les artistes de réemploi utilisent l'“archive", et la désignent comme telle, en tant que matière première de leurs films et vidéos. L'archive filmique peut être de différentes natures: il peut s'agir de films hollywoodiens, de films issus du domaine public, de films orphelins ou éphémères, mais également de films de famille, de publicités, d'émissions de télévision, de vidéos et autres films trouvés dans des brocantes ou encore dans les poubelles, c'est-à-dire tout document audiovisuel appartenant, de manière imprécise, au passé, et qui sera utilisé en partie ou dans son entièreté dans une nouvelle production.

Les archives, en tant que documents d'archives, n'apparaissent quant à elles que timidement dans la littérature. De manière générale, tout document audiovisuel semble être considéré comme un document d'archives tant par les artistes que par les chercheurs, en raison de son appartenance au passé et comme trace de ce qui a été. Les auteurs ne font que très peu référence à des textes issus des disciplines archivistique ou historique pour alimenter leur réflexion concernant les archives, si ce n'est l'ouvrage Le goût de l'archive d'Arlette Farge (1989). Des problématiques communes aux archivistes, chercheurs et autres professionnels du cinéma (notamment les cinémathèques) sont pourtant régulièrement abordées. Il s'agit principalement des questions autour de l'accès, la préservation et la conservation tant des films d'archives que des films constitués d'archives.

\section{Révolution numérique et excès archivistique}

Les mutations technologiques du monde numérique ont entraîné un changement de paradigme dans tous les domaines de recherche : les objets et sujets étudiés doivent être repensés dans la société numérique développant de nouveaux modes de pensées 
et d'agir (Compiègne 2011). Cette problématique est le moteur de questionnements dans les introductions de diverses publications récentes, notamment dans L'attrait de l'archive (Blümlinger 2014b) et dans Cinephemera. Archives, Ephemeral Cinema, and New Screen Histories in Canada (Druick et Cammaer 2014).

Du son aux images numériques et numérisées, les conservateurs et archivistes doivent faire face à une augmentation constante du nombre d'archives audiovisuelles. Les artistes de réemploi, quant à eux, ont à leur disposition un nombre toujours plus grand d'images en mouvement. Ce phénomène est qualifié d'archival excess par Catherine Russell (2013) et pose différentes questions critiques: Quels types de savoirs sont communiqués par cet excès? Quelle historiographie et quelle critique culturelle sont transmises à travers la prolifération de l'imagerie des archives? Qu'arrive-t-il à notre compréhension de l'archive? Existe-t-il une nouvelle forme d'archive? Les anciens modèles d'analyse sont-ils toujours valables? Autant de questions sur la nature des documents d'archives qui font l'écho aux préoccupations des archivistes face aux changements du numérique. Néanmoins, il est intéressant de constater que les études cinématographiques posent la question d'une éventuelle redéfinition de l'archive, là où la plupart des archivistes souhaitent au contraire la maintenir, à travers leurs fonctions et leurs valeurs, tout en reconnaissant les changements en cours.

De YouTube à la dissémination du cinéma sur Internet, cet excès archivistique entraîne un phenomenal shift au sein des archives audiovisuelles (Russell 2013). Ce changement se manifeste sous différentes formes: il est matériel, épistémologique, esthétique et économique (Druick et Cammaer 2014) et touche tant les artistes que les conservateurs et "archivistes", en passant par les chercheurs. L'archiviste et cinéaste Rick Prelinger, quant à lui, souligne également un autre type de changement, mais en nuançant son impact: "it may be a propos to characterize the great shift in archival culture as a movement from institutional to personal, but it would be foolhardy to overestimate the wisdom of crowds or underestimate the survivability of venerable institutions" (Martel et Prelinger 2013, 123).

Du point de vue des artistes de réemploi, l'accès aux documents audiovisuels est essentiel. La mise en ligne de larges collections d'images en mouvement représente une possibilité presque illimitée de création de nouveaux films. Comme le souligne l'artiste Ryan Tebo dans un article qui interroge la nature de l'archive:

As one such artist, what would be my ideal relationship with archives? Having access to as much material as possible seems as important as having an easy way to access the material. For me, establishing a fair relationship between my use and the rights of the material is a conversation that begins early in my contact with archives. (Tebo 2010)

Dans ce cadre, la question de droits d'accès, d'utilisation et de (re)diffusion des images devient fondamentale. Des initiatives indépendantes, comme l'Internet Archive (hébergeant les Archives Prelinger numérisées)² réunissent du matériel (images, films, livres, textes, sons, logiciels) libre de droits afin de le mettre à la disposition du public. Par contre, dans un geste plus critique, certains cinéastes désirent au contraire utiliser du matériel non libre de droits, afin de créer leurs films : ils vont alors chercher leurs 
matières premières sur des sites ou réseaux illégaux, certains d'entre eux entretenus par des passionnés, témoignant d'une véritable cinéphilie et d'une rigueur quant à l'archive filmique. Outre le travail de collecte, d'archivage, d'identification et parfois de restauration des films, ces sites de partage proposent des fiches de présentation des films "expliquant [l]es contextes de réalisation, de distribution ou de diffusion, et permettant ainsi d'identifier les origines de la version proposée [du film] et ses manipulations technologiques (restauration, doublage, sous-titrages, etc.) effectuées dans le cadre de la mise en ligne" (Renouard 2014, 246).

Un des défis pour les archivistes et les créateurs réside dans l'articulation de l'analogue et du numérique (Lundemo 2012). Actuellement, cette relation prend trois formes: la conversion (le numérique substitue l'analogue, mais les deux supports sont conservés et utilisés séparément), la convergence (l'analogue cède entièrement la priorité au numérique, sous une forme multimédia) ou encore la combinaison (analogue et numérique sont utilisés ensemble, entre convergence et conversion) (Lundemo 2012).

Outre les questions matérielles, esthétiques et économiques, les changements constants issus du numérique entraînent également un nouveau regard sur l'histoire des films comme archives:

... le mode de transcendance lié au numérique est celui qui ouvre au film les portes d'une "nouvelle histoire" (pour laquelle il faut probablement prévoir un statut qui ne coïncide pas avec celui de l'histoire classique), dominée par la multiplication des formes d'existence, des pratiques de transcodage et compression, et de l'intensification des rapports avec l'usager-spectateur (Quaresima 2014, 230).

\section{Historicité, temporalité et mémoire}

Dans son introduction pour le numéro de la revue Cinémas consacrée à l'attrait de l'archive, Christa Blümlinger (2014b) met l'emphase sur trois modes propres aux matériaux archivistiques: la temporalité, l'historicité et la mémoire. Il s'agit en effet de trois concepts auxquels font souvent référence les auteurs dans la théorie du cinéma de réemploi quand il est question de l'archive. En parallèle, deux modes sont également attribués aux films : la temporalité (propre aux images en mouvement) et la figurabilité (Blümlinger 2013). La structure proposée par ces différents modes propres aux deux objets, archives et films, se rencontre dans les films de réemploi. Composés d'images appartenant au passé, ces derniers s'inscrivent dans un dialogue représentatif entre passé et présent, engageant le spectateur dans son rapport au temps, à l'histoire et à la mémoire.

Dans ce cadre, les archives sont considérées comme source de l'histoire, mais également de la mémoire: "On pourrait dire que l'archive désigne ce sur quoi s'inscrit une mémoire de l'histoire (personnelle ou collective, souvent les deux à la fois)" (Habib 2008, 225). À travers ce rôle, elles deviennent alors un élément clé de la création, tant sur un plan esthétique que critique. Tout d'abord visuellement, en créant une disparité temporelle perçue comme telle par le spectateur. L'intégration d'archives dans un film crée ce que Jaimie Baron appelle l' "archive effect" (Baron 2014), une relation entre le spectateur et l'objet filmique menant à la distinction entre trois temporalités propres 
aux films faits d'archives: le "alors" du document d'archives, le "maintenant" de la production du film et le "maintenant" du visionnement du film (Baron 2014). Ensuite, les films d'archives peuvent construire une critique en problématisant le présent par le passé (Russell 2013). Les documents, tout autant que les personnes les manipulant, sont les moteurs de cette historicité révélée: les artistes en comblant les absences de l'histoire (Blümlinger 2014b) et les films comme langage qui s'adresse aux impasses de l'historicité (Russell 2013). En 1999, Russell mettait déjà l'accent sur ce point, associé à une certaine autorité apposée aux documents d'archives utilisés dans les films: "Appropriation, as an aesthetic practice, is a discourse of the uncanny, producing a figure of lack doubled by a masking of the lack. The found image doubles the bistorical real as both truth and fiction, at one document of history and unreliable evidence of bistory." (Russell 1999, 252)

La conception de l'histoire et de la mémoire dans les films de réemploi passe par la forme, les dispositifs et le matériau, ainsi que par le collage et le montage (Blümlinger 2013). Pour Habib (2008), les films expérimentaux utilisant des archives relèvent en effet des modes alternatifs d'écriture de l'histoire: ils redéfinissent la compréhension du spectateur en matière de temporalité ou d'espace filmique. Les films se situent dès lors dans un entre-deux temporel qui devient leur thème et le site de leur expérience (Habib 2008).

Dans ce cadre, la matérialité des films et des archives joue un rôle important. Les supports visuels et sonores renvoient en effet à deux types d'historicité: l'une esthétique, naissant de "la conscience d'une histoire des formes" (Blümlinger 2013, 13), l'autre culturelle "visant des formes symboliques, des contextes de l'histoire de la perception ou encore des conditions de la discursivité historique" (Blümlinger 2013, 13). D'un autre côté, si l'on soustrait cet aspect historico-narratif, le film renvoie alors à une lecture esthétique qui conduit le spectateur à une perception de pure temporalité (Blümlinger 2013).

Depuis les années 1990, l'historicité et la mémoire propres aux films faits d'archives sont mises à l'épreuve de l'excès archivistique et des mutations technologiques. Plusieurs auteurs évoquent cette inquiétude qui se manifeste sous différentes formes. D'une part, la prolifération et la surexploitation des images par les industries culturelles qui, motivées par un argument commercial, modifient les archives à l'excès, tout en les privant de leur historicité (Lindeperg 2014). D'autre part, la disponibilité sans cesse croissante des documents audiovisuels privilégiant une vision de l'archive attachée "à la circulation ou à la mise en réseau, au détriment d'une conscience des sources" (Blümlinger 2013, 8).

De manière plus alarmiste, le réalisateur Philippe Roger s'interroge : "entre sousexposition du mystère orphique et surexposition à la consommation frankensteinenne, quel avenir pour l'archive?" (Roger 2014) Il en conclut que l'immédiateté, la réactualisation et les utilisations excessives des images nient le passé auquel elles font référence et que dès lors, plus rien n'est archive dans la société actuelle. Sans toutefois les condamner de manière aussi virulente, d'autres chercheurs reconnaissent effectivement les changements apportés par le nombre croissant, l'accessibilité et l'omniprésence des images comme transformateurs de la mémoire humaine (Gagnon 2014). 


\section{Réemploi comme mode de conservation et comme archive}

La relation entre les archives et le cinéma est une relation complexe, tour à tour fascinée et angoissée, tendue entre un enthousiasme programmateur parfois destructeur et une volonté conservatrice. La fascination des images en mouvement est en effet souvent confrontée au pragmatisme des pellicules détériorées (volontairement ou non) et aux données numériques instables. Ce délicat dialogue, présent dans les préoccupations des cinémathèques, des archivistes du cinéma et chez certains cinéastes, est au cour de la problématique du cinéma de réemploi.

La dualité de cette problématique a été mise en avant par deux conservateurs. D'un côté, Henri Langlois, fondateur de la Cinémathèque française aux côtés de Georges Franju, qui pense la préservation à travers le dispositif, la projection, la programmation (Habib 2013a). De l'autre, Ernest Lindgren, premier conservateur de la National Film Archive, pour lequel la conservation passe par la sélection et la non-projection (Le Roy 2013). L'exemple des différentes politiques de conservation menées par les cinémathèques et archives de film illustre cette tension. Très souvent, c'est donc la valeur accordée aux films qui les sauve : les institutions établissent des critères d'inclusion ou d'exclusion, dépendant entre autres de leur budget et de l'espace dont elles disposent, pouvant être très strictes (Quanz 2014). De plus, les archivistes conservateurs, dans leurs gestes de sauvegarde et de conservation, peuvent être amenés à "changer le montage, [intervenir] sur la couleur et la luminosité, [nettoyer] le son, [recréer] des inserts, etc., ceci en fonction de l'état du matériel" (Lenk 2012, 78).

Cette situation de sélection engendre différentes réactions, notamment la mise en ligne de collections d'archives filmiques par des individus passionnés, professionnels ou amateurs. Un exemple illustre est celui des archives Prelinger, fondées en 1983 par Rick Prelinger, dont le but est de collecter, préserver et rendre accessible les films d'importance historique qui n'ont pas été collectés ailleurs (Prelinger Archives (s.d.)). La collection Prelinger est constituée de plus de 60000 films éphémères, c'est-à-dire des films industriels, éducatifs, amateurs et commerciaux, documentant la vie quotidienne, la culture, l'industrie et l'expérience personnelle (Martel et Prelinger 2013). Actuellement, presque 3000 de ces films sont disponibles en ligne, téléchargeables en différents formats et libres de droits. Il s'agit d'une source conséquente dans laquelle les artistes de réemploi peuvent aller chercher des images rapidement et gratuitement. Mais, si la mise en accès de la collection Prelinger représente l'initiative la plus grande en la matière, elle n'est pas la seule. Des sites communautaires de partage s'organisent autour de certains types de films, comme les films de famille (Center for Home Movies (s.d.)), autour de certains supports (VHS, par exemple) ou encore autour d'un enthousiaste cinéphile pour les films perdus, interdits ou non réédités. Rejoignant l'idée de la conservation des films de Langlois, ces initiatives rejoignent le travail des archivistes: "Les sites communautaires de partage de raretés pourraient alors être perçus comme des institutions archivistiques à part entière, de par leur travail de collecte des données, d'archivage des liens de téléchargement, mais aussi d'identification et de restaurations des œuvres." (Renouard 2014, 241)

Une seconde réflexion peut être menée à partir des politiques de conservation. Certains auteurs considèrent en effet la pratique du réemploi comme une forme de conservation des archives. André Habib évoque cet aspect unique, avec l'exemple 
de films de réemploi expérimentaux constitués d'un seul film préexistant, modifié selon certaines techniques par un cinéaste, permettant de "rendre ces premiers films à nouveau visibles et lisibles en faisant jaillir de nouveaux potentiels" (Habib 2013b, 147-148). Plus loin, il explique:

D'une certaine manière, on pourrait dire que les films de ces cinéastes sont des petits entrepôts, des lieux, des sites où l'on peut trouver des films entiers, une archive en somme bien particulière (puisque cette archive ne contient souvent qu'un seul film!), qui tout à la fois conserve et institue un mode de lecture pour ces films des "origines", à travers leur réappropriation au présent (par le cinéaste et le spectateur). (Habib 2013b, 148)

Les cinéastes de réemploi permettent donc à certains films, en leur donnant une seconde vie, d'être projetés (Cammaer 2014). Ces artistes attirent l'attention de nouveaux spectateurs sur des films qui n'ont, à un moment donné, peut-être pas ou peu d'intérêt, voire de valeur, aux yeux des conservateurs. Dès lors, ils permettent à ces films d'être conservés, en partie ou en entier: la pratique du réemploi, du found footage devient alors pratique archivistique.

\section{Collaborations entre archivistes, artistes et études cinématographiques}

Pour beaucoup d'auteurs, il est essentiel de développer une collaboration entre les différents acteurs réunis autour de l'archive que ce soit les artistes, les chercheurs, les communautés d'intérêts, les archivistes ou les cinéphiles. Car, si les pratiques et les préoccupations peuvent différer, "chacun, avec ses méthodologies et ses traditions propres, emprunte des détours uniques menant à l'archive" (Martel 2013). Cette volonté d'échange de compétences et de pratiques est d'autant plus présente que nous nous trouvons dans une période de mutations technologiques, soulevant des questions communes à travers les disciplines: "It is precisely at this time of transition that the dialogue between scholars and archivists can be particularly valuable for both theory and practice." (Fossati 2009, 103)

Souvent, il est constaté dans la littérature que peu de ponts sont construits entre les différentes disciplines et pratiques autour des archives et du cinéma. Pourtant, de nombreux points communs ont été relevés, notamment un intérêt pour la matérialité du film, des problèmes pratiques similaires et les mêmes technologies : "In other words, if there have not been many references to archival practices in film and media studies, there has always been a string (albeit unrecognised) affinity between the work of found footage filmmakers and film archivists." (Cammaer 2014)

De plus, cette collaboration est cruciale pour la compréhension tant de l'archive que des films:

... le dialogue entre les historiens du cinéma des premiers temps, les restaurateurs, les archivistes et les cinéastes expérimentaux, ce mouvement d'aller-retour entre l'intelligence historienne du passé et sa réactivation dans les œuvres artistiques, est essentiel pour éclairer notre connaissance et enrichir notre expérience de l'histoire du cinéma et, de façon plus générale, notre appréhension de ce qui se trouve dans les archives du cinéma (Habib 2013b, 151). 


\section{CONCLUSION}

À la lecture de la récente littérature au sujet du cinéma de réemploi, il est évident que les études cinématographiques et les artistes manifestent un réel intérêt pour les archives et l'archive. Différents thèmes sont évoqués : il est tour à tour question de redéfinir l'archive, de considérer la mort du film (en tant que document d'archives audiovisuel) dans sa complexité intellectuelle, esthétique et matérielle ou encore de célébrer l'attrait des ruines et l'inscription dans le temps et dans la mémoire des films faits d'archives. De ces thèmes émergent diverses problématiques, repensées à travers les mutations technologiques de la société numérique. Il est intéressant de constater que les questions générées dans ce cadre possèdent un véritable fondement archivistique, même si celui-ci n'est pas évoqué comme tel. Ainsi, différentes préoccupations communes peuvent être soulignées. Que représentent ces enjeux pour les études en archivistique?

Tout d'abord, une définition de ce que représente et constitue l'archive pour les cinéastes serait à proposer. Les différents termes utilisés varient selon les contextes, mais ne font que très rarement référence aux documents d'archives tels que considérés par les archivistes. Il serait dès lors intéressant de dresser un portrait de l'archive utilisée dans le cinéma de réemploi et de la considérer dans une perspective archivistique : de manière générale, un film peut-il être considéré comme un document d'archives? Quels types de films sont considérés comme des archives? Quelles sont les caractéristiques des documents audiovisuels et des archives dans le cinéma de réemploi?

Ensuite, une structure d'analyse commune émerge de ces lectures: il s'agit du cadre de la temporalité, de l'historicité et de la mémoire, propre aux archives et aux films. Les films faits d'archives deviennent alors des discours sur la mémoire et sur l'histoire, en empruntant des archives audiovisuelles appartenant à un certain passé, tout en s'adaptant à la temporalité propre aux matériaux filmiques. Ces trois concepts peuvent servir de terrain commun aux différentes disciplines traitant de l'archive.

Si des enjeux conjoints aux archives et au cinéma émergent à travers le réemploi, et ce tout particulièrement à l'âge numérique, on constate que le dialogue entre études archivistiques et cinématographiques est peu développé. De nombreux auteurs, tels que Prelinger, Martel, Païni, Habib et Cammaer, insistent toutefois sur le besoin de collaborations entre artistes, archivistes et chercheurs afin de développer une compréhension de l'archive et des problématiques qui en découlent (le discours étant principalement axé sur des problèmes pratiques tels que la préservation, la conservation et l'accès).

Finalement, si l'on considère que les artistes d'avant-garde et expérimentaux ont permis une redécouverte du cinéma des premiers temps, bien avant que les études cinématographiques s'y intéressent, comme le suggère André Habib (2013b), peut-on considérer que ces mêmes artistes, en s'intéressant aux archives, révèlent une autre manière de considérer les archives? Pour l'archiviste et cinéaste Rick Prelinger, il s'agit d'une possibilité à considérer: "the most interesting developments in the redefinition of our relationships to archives will arise from practices from the margins" (Martel et Prelinger 2013, 140). En effet, en s'affranchissant des contraintes professionnelles propres aux archivistes, mais également en repoussant les limites d'une lecture classique du document, ils sortent les archives de leur contexte afin de les interpréter sous un 
nouvel angle. Il s'agit "d'une occasion de montrer non seulement la richesse et la diversité des points de vue que peut engendrer l'archive, mais aussi et surtout ce qui est le propre de celle-ci : son pouvoir, sa capacité à signifier, à se révéler selon les situations" (Lemay 2009, 236-237). Si les archives sont justifiées par leur utilisation et leur exploitation, elles ont également besoin d'autre chose qu'elles-mêmes pour être activées, fécondes (Habib 2013a).

Annaëlle Winand Étudiante au doctorat, École de bibliothéconomie et des sciences de l'information, Université de Montréal.

\section{NoTES}

1. Travail réalisé à l'EBSI, Université de Montréal, sous la direction du professeur

2. https://archive.org/index.php. Yvon Lemay dans le cadre du séminaire SCI7001 - Lectures dirigées à l'hiver 2015.

\section{BIBLIOGRAPHIE}

BARON, J. 2014. The Archive Effect. Found Footage and the Audiovisual Experience of History. London, New York, Routledge.

BLÜMLINGER, C. 2013. Cinéma de seconde main. Esthétique du remploi dans l'art du film et des nouveaux médias. Traduit par Pierre Rusch et Christophe Jouanlanne. Paris, Klincksieck.

BLÜMLINGER, C. 2014a. L'attrait de plans retrouvés. Cinémas: Revue d'études cinématographiques, 24, 2-3, 69-96.

BLÜMLINGER, C. 2014b. Présentation. Cinémas: Revue d'études cinématographiques, 24, 2-3, 7-16.

CAMMAER, G. 2014. Films Collecting Dust and Dusty Film Collages. Ephemerality at Work. In Cinephemera. Archives, Ephemeral Cinema, and New Screen Histories in Canada, sous la dir. de Z. Druick et G. Cammaer. Montréal, McGill-Queen's University Press.

CENTER FOR HOME MOVIES. (s.d.). Center for Home Movies. [En ligne]. http://www. centerforhomemovies.org/ (Page consultée en 2015).

COMPIÈGNE, I. 2011. La société numérique en question(s). Auxerre, Éditions Sciences Humaines.

DERRIDA, J. 1995. Mal d'archive: une impression freudienne. Paris, Galilée.

DRUICK, Z. et G. CAMMAER. 2014. Introduction : Canadian Cinema, Ephemeral Cinema. Dans Z. Druick et G. Cammaer (dir.), Cinephemera. Archives, Ephemeral Cinema, and New Screen Histories in Canada (p. 3-13). Montreal \& Kingston, McGillQueen's University Press.

FARGE, A. 1989. Le goût de l'archive. Paris, Éditions du Seuil. 
FOSSATI, G. 2009. From Grain to Pixel The Archival Life of Film in Transition. Amsterdam, Amsterdam University Press.

FOUCAULT, M. 1969. L'archéologie du savoir. Paris, Gallimard.

GAGNON, M. K. 2014. Unfinished Films and Posthumous Cinema. Charles Gagnon's R69 and Joyce Wiedland's Wendy and Joyce. Dans Z. Druick et G. Cammaer (dir.), Cinephemera. Archives, Ephemeral Cinema, and New Screen Histories in Canada (p. 137-158). Montreal \& Kingston, McGill-Queen's University Press.

HABIB, A. 2008. Le temps décomposé: cinéma et imaginaire de la ruine. (Thèse de doctorat, Université de Montréal). Repéré à http://hdl.handle.net/1866/6641.

HABIB, A. 2013a. Cinéma : l'invention du musée. Entretien avec Dominique Païni. Dans M. Marie et A. Habib (dir.), L'avenir de la mémoire. Patrimoine, restauration, réemploi cinématographiques (p. 17-24). Villeuneuve d'Ascq, Presses Universitaires du Septentrion.

HABIB, A. 2013b. Le cinéma de réemploi considéré comme une "archive". L'exemple de A Trip Down Market Street (1906) et Eureka (1974). Dans M. Marie et A. Habib (dir.), L'avenir de la mémoire. Patrimoine, restauration, réemploi cinématographiques (p. 147-158). Villeuneuve d'Ascq, Presses Universitaires du Septentrion.

HABIB, A. 2014. Archives, modes de réemploi. Pour une archéologie du found footage. Cinémas: Revue d'études cinématographiques, 24(2-3), 97. doi :10.7202/1025150ar.

KLEIN, A. 2015. Archive(s): approche dialectique et exploitation artistique. (Thèse de doctorat, Université de Montréal). Repéré à http://hdl.handle.net/1866/11648.

LE ROY, É. 2013. Cinémathèques et archives du film. Paris: Armand Colin.

LEMAY, Y. 2009. Le détournement artistique des archives. Dans P. Servais, F. Hiraux et F. Mirguet (dir.), Les maltraitances archivistiques. Falsifications, instrumentalisations, censures, divulgations (p. 223-240). Louvain-la-Neuve, Academia Bruylant.

LENK, S. 2012. Le film, un "artifact archival"? Dans A. Bordina, S. Campanini, et A. Mariani (dir.), L'archivio. Atti del XVIII Convegno internazionale di studi sul cinema, Udine, 5-7 aprile 2011 (p. 77-85). Udine, Forum.

LEYDA, J. 1964. Films beget films. Londres, George Allen \& Unwin Ltd.

LINDEPERG, S. 2014. La voie des images. Valeur documentaire, puissance spectrale. Cinémas: Revue d'études cinématographiques, 24(2-3), 41-68. doi :10.7202/1025148ar.

LUNDEMO, T. 2012. Conversion, Convergence, Conflation; Archival Networks in the Digital Turn. Dans A. Bordina, S. Campanini, et A. Mariani (dir.), L'archivio. Atti del XVIII Convegno internazionale di studi sul cinema, Udine, 5-7 aprile 2011 (p. 177-182). Udine, Forum.

MARTEL, C. 2013. "It's not gone, it's just hiding". Quelques notes de recherche-création avec les films orphelins. Dans M. Marie et A. Habib (dir.), L'avenir de la mémoire. Patrimoine, restauration, réemploi cinématographiques (p. 135-145). Villeuneuve d'Ascq, Presses Universitaires du Septentrion. 
MARTEL, C. et R. PRELINGER. 2013. Looking Back at an Electronic Exchange with a Media Archeologist. An interview with Rick prelinger by Caroline Martel 12 1/2 years later. Dans M. Marie et A. Habib (dir.), L'avenir de la mémoire. Patrimoine, restauration, réemploi cinématographiques (p. 115-123). Villeuneuve d'Ascq, Presses Universitaires du Septentrion.

PRELINGER ARCHIVES. (s.d.). Prelinger Archives : Collections Summary. Repéré à http:// www.prelinger.com/collections_summary.html.

QUANZ, K. 2014. Preserving Ephemeral Aboriginal Films and Videos. The Archival Practices of Vtape and ISUMATV. Dans Z. Druick et G. Cammaer (dir.), Cinephemera. Archives, Ephemeral Cinema, and New Screen Histories in Canada (p. 256-272). Montréal, McGill-Queen's University Press.

QUARESIMA, L. 2014. Du cimetière des éléphants au parc thématique? L'archive à l'époque de la numérisation. Dans G. Pisano (dir.), L'archive-forme. Création, mémoire, bistoire (p. 218-237). Paris, L'Harmattan.

RENOUARD, C. 2014. Les "pirates" du web : archives de films rares et réseaux illégaux. Dans G. Pisano (dir.), L'archive-forme. Création, mémoire, histoire (p. 239-249). Paris, L'Harmattan.

ROGER, P. 2014. Les enjeux de l'archive filmée : positions et propositions (à propos du documentaire Le récital de Besançon). Dans G. Pisano (dir.), L'archive-forme. Création, mémoire, histoire (p. 93-104). Paris, L'Harmattan Editions Distribution.

RUSSELL, C. 1999. Experimental Ethnography. The Work of Film in the Age of Video. Duke University Press.

RUSSELL, C. 2013. Benjamin, Prelinger, and The Moving Image Archive. Dans M. Marie et A. Habib (dir.), L'avenir de la mémoire. Patrimoine, restauration, réemploi cinématographiques (p. 101-113). Villeuneuve d'Ascq, Presses Universitaires du Septentrion.

TEBO, R. 2010. What is an Archive, What Does it Do, and What/Who is it Good For? Incite!, (2). Repéré à http://www.incite-online.net/tebo2.html.

TORLASCO, D. 2013. The Heretical Archive. Digital Memory at the End of Film. Minneapolis, University of Minnesota Press.

WEES, W. C. 1993. Recycled Images. The Art and Politics of Found Footage Films. New York, Anthology Film Archives.

WEES, W. C. 2014. Breaking New Ground. Canada's First Found Footage Films. Dans Z. Druick et G. Cammaer (dir.), Cinephemera. Archives, Ephemeral Cinema, and New Screen Histories in Canada (p. 112-136). Montréal \& Kingston, McGillQueen's University Press. 